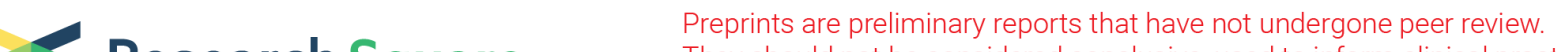 Research Square
or referenced by the media as validated information.
}

\section{Clinical Analysis of Gastrointestinal Bleeding in Patients with Severe Coronavirus Disease (COVID- 19): A Retrospective Observational Study}

Jinfen Dai

Tongji Hospital of Tongji Medical College of Huazhong University of Science and Technology

Song $\mathrm{Hu}$

Tongji Hospital of Tongji Medical College of Huazhong University of Science and Technology Yi Chen

Tongji Hospital of Tongji Medical College of Huazhong University of Science and Technology

Pengbo Wu

Renmin Hospital of Wuhan University: Wuhan University Renmin Hospital

Mei Liu ( $D$ liumei20200503@126.com )

Tongji Hospital of Tongji Medical College of Huazhong University of Science and Technology

Research article

Keywords: gastrointestinal bleeding, SARS-CoV-2, COVID-19

Posted Date: September 22nd, 2020

DOl: https://doi.org/10.21203/rs.3.rs-79594/v1

License: (9) This work is licensed under a Creative Commons Attribution 4.0 International License.

Read Full License 


\section{Abstract}

Background: The epidemic of Novel coronavirus disease 2019(COVID-19) has attracted the great attention of people all over the world. Gastrointestinal bleeding in patients with COVID-19 has been studied rarely. We mean to investigate the clinical characteristics of gastrointestinal bleeding in patients with severe coronavirus disease (COVID-19).

Methods: Nineteen severe COVID-19 patients with gastrointestinal bleeding were retrospectively studied. Clinical data, including medical history, symptoms, laboratory characteristics, and treatment, were collected and analyzed. We also compared the characteristics of patients with different degrees of bleeding.

Results: Fourteen patients $(73.6 \%)$ were men. Mean age was $63.8( \pm 15.6)$ years. Most patients $(89.4 \%)$ had no history of gastrointestinal bleeding. The most common presentation of gastrointestinal bleeding was a positive fecal occult blood test (15/19 cases, $78.9 \%)$. Fifteen patients (78.9\%) did not require blood transfusion. Five of 15 patients $(33 \%)$ received anticoagulants and antiplatelet drugs before gastrointestinal bleeding in the mild bleeding group, and 2/4 (50\%) in the severe bleeding group. Four of 15 patients $(27 \%)$ died in the mild bleeding group and no patients died in the severe bleeding group.

Conclusions: The presentations of gastrointestinal bleeding in severe COVID-19 patients are not obvious, and frequent fecal occult blood test is needed to detect gastrointestinal bleeding early. Patients receiving anticoagulants and antiplatelet drugs need a close observation for gastrointestinal bleeding. Gastrointestinal bleeding may not increase the mortality, after giving blood transfusion support therapy.

\section{Background}

A novel coronavirus named 2019-nCoV, also identified as the severe acute respiratory syndrome coronavirus 2 (SARS-CoV-2) [1], which caused an outbreak of unexplained pneumonia in Wuhan, China, has spread rapidly across the world since December 2019. The number of people with coronavirus disease (COVID-19) has been increasing rapidly. There have been 83,745 confirmed cases in China and $1,904,587$ confirmed cases in the rest of the world as of April 15,2020. Considering that even children and pregnant women are generally susceptible to COVID-19 [2] and that there have been no specific drugs or vaccines [3, 4], COVID-19 emerged as a great threat to public health. Similar to SARS-CoV, SARS-CoV-2 causes severe respiratory syndrome by entering the cells through the receptor angiotensin-converting enzyme $2[2,5]$. Apart from respiratory symptoms, the symptoms of COVID-19 include gastrointestinal complaints, such as loss of appetite, nausea, vomiting, diarrhea, and abdominal pain [6].

Although it seems that most COVID-19 infections are mild, a minority of patients become severely ill as the disease progresses. Gastrointestinal bleeding is one of the critical digestive diseases, requiring great attention in clinical practice. It also occurs in patients with COVID-19, especially in severe cases, which needs more attention and special care. Nevertheless, there have been few studies on gastrointestinal 
bleeding in COVID-19 patients. In this study, we aimed to analyze the clinical characteristics of gastrointestinal bleeding in patients with severe COVID-19 to ensure better prevention and treatment.

\section{Methods}

\section{Patients and data collection}

This study included a total of 19 patients with severe COVID-19 with gastrointestinal bleeding, who were admitted to Tongji Hospital, Tongji Medical College, Huazhong University of Science and Technology from February 10 to March 29, 2020. The diagnosis of severe COVID-19 was based on the WHO interim guidance, diagnosis of pneumonia, and treatment scheme for novel coronavirus infection (trial version 6) issued by National Health Commission of China [7]. Our study was approved by the Medical Ethics Board of the Tongji Hospital, Tongji Medical College, Huazhong University of Science and Technology.

We defined gastrointestinal bleeding based on clinical symptoms, including hematemesis, bloody stools, and melena, or positive fecal occult blood test. The baseline characteristics of patients, including demographic data, symptoms, comorbidities, and medical history, as well as laboratory and treatment data, were collected and analyzed.

\section{Statistical analysis}

Continuous data were presented as mean \pm standard deviation (SD). $T$ test was used to assess the differences between the groups. Categorical variables were presented as frequencies. Statistical analysis was performed using SPSS 17.0. The $p$ value of less than 0.05 was considered to be significant.

\section{Results}

\section{Baseline characteristics}

Fourteen of 19 patients $(73.6 \%)$ were men, and $5(26.3 \%)$ were women. The mean age was $63.8( \pm 15.6)$ years. Fever and cough were the most common initial symptoms, including four patients $(21.1 \%)$ with fever, two (10.5\%) with cough, and five (26.3\%) with both fever and cough. There were also single cases of dyspnea, bloody stool, both fatigue and anorexia, both cough and dyspnea, both fever and dyspnea, both fever and diarrhea, both fever and myalgia, and no symptoms $(n=1,5.3 \%$ each). The most common comorbidity was hypertension (26.3\%). Seven patients (36.8\%) had more than one comorbidity. There were single patients with one of the following comorbidities or combinations of comorbidities: diabetes, both hypertension and cerebrovascular disease, both hypertension and pulmonary disease, both hypertension and cardiovascular disease, both hypertension and diabetes, both hypertension and benign prostatic hyperplasia, three diseases (hypertension, diabetes, and cerebrovascular disease; or hypertension, cardiovascular disease, and chronic renal failure). Six patients (31.6\%) had no comorbidities. Most patients (89.4\%) had no prior history of gastrointestinal bleeding. One patient (5.3\%) had a peptic ulcer hemorrhage one year ago, and one patient had prior gastrorrhagia (Table 1). 
Table 1

Baseline characteristics of COVID-19 patients with gastrointestinal bleeding.

\begin{tabular}{|l|l|}
\hline Clinical characteristics & $\mathbf{N}(\%)$ \\
\hline Gender & \\
\hline Male & $14(73.7 \%)$ \\
\hline Female & $5(26.3 \%)$ \\
\hline Age (mean \pm SD) [years] & $63.8 \pm 15.6$ \\
\hline Initial symptoms & \\
\hline Fever & $4(21.1 \%)$ \\
\hline Cough & $2(10.5 \%)$ \\
\hline Dyspnea & $1(5.3 \%)$ \\
\hline Bloody stool & $1(5.3 \%)$ \\
\hline Both fatigue and anorexia & $1(5.3 \%)$ \\
\hline Both fever and cough & $5(26.3 \%)$ \\
\hline Both cough and dyspnea & $1(5.3 \%)$ \\
\hline Both fever and dyspnea & $1(5.3 \%)$ \\
\hline Both fever and diarrhea & $1(5.3 \%)$ \\
\hline Both fever and myalgia & $1(5.3 \%)$ \\
\hline No symptoms & $1(5.3 \%)$ \\
\hline Comorbidities & $1(51.6 \%)$ \\
\hline Hypertension & $1(5.3 \%)$ \\
\hline Diabetes & $1(5.3 \%)$ \\
\hline Hypertension and cerebrovascular disease & $1(5.3 \%)$ \\
\hline Hypertension and pulmonary disease & $1(5.3 \%)$ \\
\hline Hypertension, diabetes, and cerebrovascular disease & $1(5.3 \%)$ \\
\hline Hypertension and cardiovascular disease & $1(5.3 \%)$ \\
\hline Hypertension and diabetes & $1(5.3 \%)$ \\
\hline Hypertension, cardiovascular disease, and chronic renal failure & $1(5)$ \\
\hline Hypertension and benign prostatic hyperplasia & \\
\hline No comorbidities & $1(5.3)$ \\
\hline
\end{tabular}




\begin{tabular}{|ll|}
\hline Clinical characteristics & $\mathbf{N}(\%)$ \\
\hline Past history of gastrointestinal bleeding & \\
\hline Peptic ulcer hemorrhage one year ago & $1(5.3 \%)$ \\
\hline Gastrorrhagia & $1(5.3 \%)$ \\
\hline Without history of gastrointestinal bleeding & $17(89.4 \%)$ \\
\hline
\end{tabular}

\section{Laboratory characteristics}

Laboratory data of severe COVID-19 patients with gastrointestinal bleeding are summarized in Table 2 . Laboratory findings showed that blood cell counts (white blood cells, neutrophils, lymphocytes, and platelets), hepatic markers (alanine aminotransferase, aspartate aminotransferase, total bilirubin, direct bilirubin, and albumin), renal function markers (blood urea nitrogen, and creatinine), and coagulation function indicators (prothrombin time, activated partial thromboplastin time, and D-dimer) did not differ between the admission day and the first day of gastrointestinal bleeding after admission $(p>0.05)$. The level of hemoglobin tested on the first day of gastrointestinal bleeding after admission was significantly reduced compared with that on admission $(p=0.004)$. 
Table 2

Laboratory characteristics of severe COVID-19 patients with gastrointestinal bleeding.

\begin{tabular}{|c|c|c|c|c|}
\hline \multirow[t]{2}{*}{ Laboratory findings } & \multirow{2}{*}{$\begin{array}{l}\text { Normal } \\
\text { range }\end{array}$} & \multicolumn{2}{|l|}{ Patients } & \multirow[t]{2}{*}{$P^{*}$} \\
\hline & & I & ॥ & \\
\hline White blood cells (WBC, $\left.\times 10^{9} / \mathrm{L}\right)$ & $3.5-9.5$ & $7.51 \pm 4.53$ & $8.8 \pm 4.3$ & 0.348 \\
\hline Neutrophils $\left(\times 10^{9} / \mathrm{L}\right)$ & $1.8-6.3$ & $5.88 \pm 4.35$ & $6.7 \pm 4.2$ & 0.541 \\
\hline Lymphocytes $\left(\times 10^{9} / \mathrm{L}\right)$ & $1.1-3.2$ & $1.01 \pm 0.46$ & $1.3 \pm 0.7$ & 0.14 \\
\hline Hemoglobin (HGB, g/L) & $115-150$ & $129.3 \pm 19.6$ & $109.7 \pm 19.9$ & 0.004 \\
\hline Platelets (PLT, $\left.\times 10^{9} / \mathrm{L}\right)$ & $125-350$ & $208.4 \pm 88.4$ & $\begin{array}{l}217.1 \pm \\
122.7\end{array}$ & 0.803 \\
\hline Alanine aminotransferase (ALT, U/L) & $\leq 33$ & $27 \pm 18.3$ & $29.7 \pm 16.5$ & 0.632 \\
\hline Aspartate aminotransferase (AST, U/L) & $\leq 32$ & $29 \pm 14.8$ & $54.7 \pm 133.0$ & 0.408 \\
\hline Total bilirubin (TBIL, $\mu \mathrm{mol} / \mathrm{L})$ & $\leq 21$ & $10.9 \pm 4.5$ & $11.7 \pm 6.9$ & 0.66 \\
\hline Direct bilirubin (DBIL, $\mu \mathrm{mol} / \mathrm{L}$ ) & $\leq 8$ & $5.0 \pm 2.5$ & $5.6 \pm 3.2$ & 0.54 \\
\hline Albumin (ALB, g/L) & $35-52$ & $34.8 \pm 4.4$ & $34.9 \pm 5.4$ & 0.974 \\
\hline Blood urea nitrogen (BUN, mmol/L) & $2.6-7.5$ & $7.6 \pm 5.6$ & $10.5 \pm 8.2$ & 0.205 \\
\hline Creatinine (Cre, $\mu \mathrm{mol} / \mathrm{L})$ & $45-84$ & $\begin{array}{l}130.7 \pm \\
215.9\end{array}$ & $112.8 \pm 121$ & 0.754 \\
\hline Prothrombin time (PT, s) & $11.5-14.5$ & $13.9 \pm 1.4$ & $13.7 \pm 1.0$ & 0.635 \\
\hline $\begin{array}{l}\text { Activated partial thromboplastin time (APTT, } \\
\text { s) }\end{array}$ & $29-42$ & $40.2 \pm 5.6$ & $39.8 \pm 5.6$ & 0.849 \\
\hline D-dimer $(\mu \mathrm{g} / \mathrm{mL})$ & $<0.5$ & $3.4 \pm 5.2$ & $3.0 \pm 3.8$ & 0.793 \\
\hline
\end{tabular}

\section{Characteristics of gastrointestinal bleeding}

There were 5 patients with gastrointestinal bleeding on admission, while 14 other cases experienced gastrointestinal bleeding after admission, with the length of hospital stay for $13.2 \pm 13.0$ days. Most patients (84.2\%) had no obvious concomitant digestive symptoms during gastrointestinal bleeding. There was a single case $(5.3 \%)$ of abdominal pain and two cases $(10.5 \%)$ of diarrhea during gastrointestinal bleeding. The most common presentation of gastrointestinal bleeding was a positive fecal occult blood test (15/19 cases, $78.9 \%)$. One patient (5.3\%) presented with melena and three (15.8\%) presented with bloody stools. In terms of the amount of bleeding, 15 patients $(78.9 \%)$ were in the range of $5-10 \mathrm{~mL} /$ day, 
$1(5.3 \%)$ in the range of $10-50 \mathrm{~mL} /$ day, $1(5.3 \%)$ in the range of $50-100 \mathrm{~mL} /$ day, and $2(10.5 \%)$ in the range of $100-150 \mathrm{~mL} /$ day. The duration of gastrointestinal bleeding was $6.4 \pm 6.5$ days. Fifteen of 19 patients (78.9\%) did not require blood transfusion after gastrointestinal bleeding. Seven patients (36.8\%) received anticoagulants and/or antiplatelet drugs before gastrointestinal bleeding. There were five patients $(26.3 \%)$ treated with glucocorticoids before gastrointestinal bleeding (Table 3). 
Table 3

Characteristics of gastrointestinal bleeding in patients with severe COVID-19.

\section{Characteristics of gastrointestinal bleeding}

Presentation of gastrointestinal bleeding

Melena

$1(5.3 \%)$

Bloody stools

$3(15.8 \%)$

Positive fecal occult blood test

$15(78.9 \%)$

Concomitant digestive symptoms during gastrointestinal bleeding

Abdominal pain

$1(5.3 \%)$

Diarrhea

$2(10.5 \%)$

No symptoms

$16(84.2 \%)$

Amount of bleeding ( $\mathrm{mL} /$ day)

5-10

$10-50$

$1(5.3 \%)$

$50-100$

$1(5.3 \%)$

$100-150$

$2(10.5 \%)$

Length of hospital stay before

gastrointestinal bleeding (days)

$13.2 \pm 13.0$

Duration of gastrointestinal bleeding (days)

$6.4 \pm 6.5$

Transfusion

Yes

$4(21.1 \%)$

No

$15(78.9 \%)$

Treatment with anticoagulants and/or antiplatelet drugs before gastrointestinal bleeding

Yes

No

Treatment with glucocorticoids

Before gastrointestinal bleeding

Yes

$5(26.3 \%)$

No 
To improve treatment guidelines and understand the prognosis of gastrointestinal bleeding in COVID-19 patients, we divided the patients into two groups according to the amount of bleeding and compared the characteristics of patients with mild bleeding and severe bleeding. Mild bleeding group encompassed patients with positive fecal occult blood test, while severe bleeding group included patients with melena or bloody stools. Five of 15 patients (33\%) received anticoagulants and antiplatelet drugs before gastrointestinal bleeding in the mild bleeding group, compared with 2/4 (50\%) in the severe bleeding group. Four of 15 patients (27\%) died in the mild bleeding group, but no patients died in the severe bleeding group. All surviving patients were discharged. The two groups did not differ in proportion of patients with previous glucocorticoid treatment before gastrointestinal bleeding (Table 4).

Table 4

Characteristics of COVID-19 patients with different degrees of gastrointestinal bleeding.

\begin{tabular}{|lll|}
\hline & $\begin{array}{l}\text { Positive fecal } \\
\text { occult blood test } \\
\text { N (\%) }\end{array}$ & $\begin{array}{l}\text { Melena or } \\
\text { bloody stools } \\
\text { N (\%) }\end{array}$ \\
\hline History of gastrointestinal bleeding & $2(13 \%)$ & 0 \\
\hline $\begin{array}{l}\text { Treatment with anticoagulants and/or antiplatelet drugs } \\
\text { before gastrointestinal bleeding }\end{array}$ & $5(33 \%)$ & $2(50 \%)$ \\
\hline $\begin{array}{l}\text { Treatment with glucocorticoid before gastrointestinal } \\
\text { bleeding }\end{array}$ & $4(27 \%)$ & $1(25 \%)$ \\
\hline Outcome (death) & $4(27 \%)$ & 0 \\
\hline
\end{tabular}

\section{Discussion}

Recent studies have reported that some gastrointestinal symptoms, such as diarrhea, vomiting, nausea, and abdominal pain, can be found in patients with COVID-19, as a less common manifestation $[6,8,9]$. However, hitherto, gastrointestinal bleeding has not been reported in COVID-19 patients. Our study showed that gastrointestinal bleeding can be found in a proportion of patients with severe COVID-19. Gastrointestinal bleeding in patients with severe COVID-19 occurred usually during the course of disease, suggesting that gastrointestinal bleeding may also be a manifestation of COVID-19, although not one of the initial symptoms. In our study, the presentations of gastrointestinal bleeding were not obvious, and in most patients, gastrointestinal bleeding was found only by fecal occult blood test. Our findings imply that frequent fecal occult blood test is essential for timely detection of gastrointestinal bleeding in patients with COVID-19, especially in severe patients, to prevent acute aggravation.

Some previous studies discovered that COVID-19 acts on angiotensin-converting enzyme 2 (ACE2) [10, 11]. ACE2 is expressed not only in the lungs, but also in the small-intestine enterocytes, crypt cells, and the colon, which may cause inflammation susceptibility of the gastrointestinal tract $[12,13]$. In addition, anticoagulants, antiplatelet drugs, and glucocorticoids are often used in severe forms of COVID-19, which puts the patients to a higher risk of gastrointestinal bleeding. Although most of the gastrointestinal 
bleeding was mild in our study, it is important for first-line clinicians to take measures in advance to prevent massive gastrointestinal bleeding.

Gastrointestinal bleeding encompasses various forms of bleeding from the oral cavity to the rectum. A small amount of bleeding can only be detected by a fecal occult blood test. When a large amount of blood is lost in a short period of time, symptoms may include vomiting red blood, vomiting black blood, bloody stools, or black stools, which may be life-threatening. In our cases, patients with black stool and bloody stools were given blood transfusion.

We compared the characteristics between mild bleeding patients and severe bleeding patients, and tracked their final outcomes. The outcome was mainly survival or death. No statistical analysis was performed because of the small sample size. We found that the proportion of patients using anticoagulants drugs and antiplatelet drugs was slightly higher in the severe bleeding group. This reminds us that great attention to gastrointestinal bleeding is needed when using anticoagulants and/or antiplatelet drugs in patients with COVID-19. In contrast, the use of glucocorticoids and history of gastrointestinal bleeding were similar in both groups. In terms of prognosis, all of the patients in the severe bleeding group recovered, while the patients who died were in the mild bleeding group. Our research suggested that blood transfusion support therapy prevented mortality of patients with severe gastrointestinal bleeding. However, the sample size is limited and more data is needed.

This study had several limitations. The sample size was small and there may be bias, so a larger, multicenter clinical study is needed. In this study, patients did not undergo endoscopy to determine the cause of bleeding. Endoscopy is generally used to diagnose and treat hospitalized gastrointestinal bleeding patients; however, the endoscopy in COVID-19 patients with gastrointestinal bleeding puts the endoscopy staff and anesthesia staff at a high exposure to the virus $[14,15]$. In the early stage of the pandemic, protection of staff was limited, and gastrointestinal examination could not be performed. It is recommended that, in the future, COVID-19 patients with obvious gastrointestinal bleeding should undergo gastrointestinal examination to determine the cause of gastrointestinal bleeding if body condition can tolerate it.

\section{Conclusion}

Gastrointestinal bleeding may be a manifestation of COVID-19 in some patients. Gastrointestinal bleeding in patients with severe COVID-19 often occurs after a period of time from the onset of COVID-19. The presentations of gastrointestinal bleeding are not obvious, and frequent fecal occult blood test is needed to early detect gastrointestinal bleeding to prevent rapid deterioration. Patients receiving anticoagulants and/or antiplatelet drugs should be monitored for gastrointestinal bleeding. Gastrointestinal bleeding does not increase mortality if blood transfusion support therapy is given.

\section{Abbreviations}


COVID-19: coronavirus disease 2019

SARS-CoV-2: severe acute respiratory syndrome coronavirus 2

ACE2: angiotensin-converting enzyme 2

\section{Declarations}

\section{Acknowledgements}

Not applicable.

\section{Authors' contributions}

Jinfen Dai drafted the article and performed data analysis, Song Hu was responsible for data collection and drafting the article, Yi Chen performed data analysis/interpretation, Pengbo Wu performed statistical analysis, and Mei Liu was responsible for concept/design, revision, and approval of the article.

\section{Funding}

This research did not receive any specific grant from funding agencies in the public, commercial, or notfor-profit sectors.

\section{Availability of data and materials}

All data generated or analysed during this study are included in this published article.

\section{Ethics approval and consent to participate}

This study was approved by the Medical Ethics Board of the Tongji Hospital, Tongji Medical College, Huazhong University of Science and Technology.

\section{Consent for publication}

Not applicable.

\section{Competing interests}

The authors declare that they have no competing interest.

\section{References}

1. The L.Emerging understandings of 2019-nCoV. The Lancet. 2020; 395(10221):311.

2. Huang X, Wei F, Hu L, Wen L, Chen K. Epidemiology and Clinical Characteristics of COVID-19. Arch Iran Med. 2020; 23(4):268-71. 
3. Wang W, Tang J, Wei F. Updated understanding of the outbreak of 2019 novel coronavirus (2019nCoV) in Wuhan, China. J Med Virol. 2020; 92(4):441-7.

4. Feng G, Zheng KI, Yan QQ, Rios RS, Targher G, Byrne CD, et al. COVID-19 and Liver Dysfunction: Current Insights and Emergent Therapeutic Strategies. J Clin TransI Hepatol. 2020; 8(1):18-24.

5. Huang C, Wang Y, Li X, Ren L, Zhao J, Hu Y, et al. Clinical features of patients infected with 2019 novel coronavirus in Wuhan, China. The Lancet. 2020; 395(10223):497-506.

6. Luo S, Zhang X, Xu H. Don't overlook digestive symptoms in patients with 2019 novel coronavirus disease (COVID-19). Clin Gastroenterol Hepatol. 2020; 18(7):1636-37.

7. China tNHCo. Interpretation of pneumonia diagnosis and treatment scheme for novel coronavirus infection (trial version 6) issued by National Health Commission of China, http://www.nhc.gov.cn/yzygj/s7652m/202002/54e1ad5c2aac45c19eb541799bf637e9.shtml; 2020[accessed on 19 February 2020].

8. Chen N, Zhou M, Dong X, Qu J, Gong F, Han Y, et al. Epidemiological and clinical characteristics of 99 cases of 2019 novel coronavirus pneumonia in Wuhan, China: a descriptive study. Lancet. 2020; 395(10223):507-13.

9. Wang D, Hu B, Hu C, Zhu F, Liu X, Zhang J, et al. Clinical Characteristics of 138 Hospitalized Patients With 2019 Novel Coronavirus-Infected Pneumonia in Wuhan, China. JAMA. 2020; 323(11):1061-9.

10. Zhou P, Yang XL, Wang XG, Hu B, Zhang L, Zhang W, et al. A pneumonia outbreak associated with a new coronavirus of probable bat origin. Nature. 2020; 579(7798):270-3.

11. Lu R, Zhao X, Li J, Niu P, Yang B, Wu H, et al. Genomic characterisation and epidemiology of 2019 novel coronavirus: implications for virus origins and receptor binding. Lancet. 2020; 395(10224):56574.

12. Zhang H, Li HB, Lyu JR, Lei XM, Li W, Wu G, et al. Specific ACE2 Expression in Small Intestinal Enterocytes may Cause Gastrointestinal Symptoms and Injury after 2019-nCoV Infection. Int J Infect Dis. 2020; 96:19-24.

13. Hashimoto T, Perlot $T$, Rehman A, Trichereau J, Ishiguro H, Paolino M, et al. ACE2 links amino acid malnutrition to microbial ecology and intestinal inflammation. Nature. 2012; 487(7408):477-81.

14. Han J, Wang Y, Zhu L, Cui Y, Li L, Zeng Z, et al. Preventing the spread of COVID-19 in digestive endoscopy during the resuming period: meticulous execution of screening procedures. Gastrointest Endosc. 2020; 92(2):445-7.

15. Castro Filho EC, Castro R, Fernandes FF, Pereira G, Perazzo H. Gastrointestinal endoscopy during the COVID-19 pandemic: an updated review of guidelines and statements from international and national societies. Gastrointest Endosc. 2020; 92(2):440-5. 